\title{
Paediatric population pharmacokinetic and pharmacodynamic modelling of ambrisentan in pulmonary arterial hypertension and comparison with adult data
}

\author{
Malek Okour ${ }^{1}$, Mita Thapar ${ }^{2}$, Colm Farrell ${ }^{3}$, Mary Ann Lukas ${ }^{4}$, Maurice Beghetti ${ }^{5}$, and \\ Misba Beerahee ${ }^{4}$ \\ ${ }^{1}$ GlaxoSmithKline \\ ${ }^{2}$ ICON Early Phase Services \\ ${ }^{3}$ ICON Development Solutions \\ ${ }^{4} \mathrm{GSK}$ \\ ${ }^{5}$ Children's Hospital, University of Geneva
}

May 25, 2021

\begin{abstract}
Aims: To develop a population pharmacokinetic (PK) model of ambrisentan in paediatric patients aged 8 to $<18$ years with pulmonary arterial hypertension (PAH), compare paediatric ambrisentan systemic exposure to historical adult data, and assess PK-PD relationships. Methods: A previously developed adult population PK model provided an initial step for modelling the 211 PK observations from 39 paediatric patients with PAH in the randomised Phase IIb study AMB112529 (NCT01332331). Subsequently, a population PK model was developed using only paediatric PK data. Steady-state systemic exposure metrics were estimated for the paediatric population and compared with historical adult data (adult patients with PAH and healthy volunteers). Exploratory exposure-response analysis assessed ambrisentan systemic exposure versus change from baseline in 6minute walking distance in paediatric patients; findings were compared with adult data. An exploratory analysis of ambrisentan exposure versus incidence of ambrisentan-related adverse events in paediatric patients was also performed. Results: The final paediatric population PK model was a two-compartment model which includes the effect of body weight (allometric scaling), first-order absorption and elimination, and absorption lag time. Similar steady-state ambrisentan exposure was confirmed in paediatric patients and historical adult data when differences in body weight were accounted for. There was no apparent correlation in the paediatric or adult population between ambrisentan exposure and change in 6-minute walking distance, or between ambrisentan exposure and incidence of ambrisentan-related adverse events in paediatric patients. Conclusions: Similar ambrisentan exposure and PK-PD profiles were observed in paediatric and adult populations with PAH.
\end{abstract}

\section{Hosted file}

Study 112529 ambrisentan PK paeds manuscript_20May2021_NoEN.docx available at https: //authorea.com/users/415559/articles/523409-paediatric-population-pharmacokineticand-pharmacodynamic-modelling-of-ambrisentan-in-pulmonary-arterial-hypertension-andcomparison-with-adult-data 
figures/GSK-[7005]-Fig-01/GSK- [7005]-Fig-01-eps-converted-to.pdf 
figures/GSK-[7005]-Fig-02/GSK-[7005]-Fig-02-eps-converted-to.pdf 
figures/GSK-[7005]-Fig-03/GSK-[7005]-Fig-03-eps-converted-to.pdf 
figures/GSK-[7005]-Fig-04/GSK- [7005]-Fig-04-eps-converted-to.pdf 
figures/GSK-[7005]-Fig-05/GSK-[7005]-Fig-05-eps-converted-to.pdf 